\title{
Electrical and Mechanical Design of a Vest Measuring a Large Set of Physiological Signals
}

\author{
Josias Wacker, Olivier Chételat, Michaël Rapin, Christophe \\ Meier, Jacques-André Porchet \\ CSEM \\ Neuchâtel, Switzerland \\ jwr@csem.ch \\ Y. L. Chang \\ Croydon University Hospital \\ Croydon, United Kingdom
}

\begin{abstract}
This paper presents the mechanical and electrical design of a wearable vest with embedded sensors that allows measuring a big number of various physiological signals. The system paves the way for closely monitoring patients with chronic obstructive lung disease in their everyday life.
\end{abstract}

Keywords-sensors; EIT; ECG; wearable; COPD

\section{INTRODUCTION}

COPD (Chronic Obstructive Pulmonary Disease) is a disease in which the airflow into the lungs and back is hindered as a consequence of exposure to irritants (e.g., smoke) causing an inflammatory response in the respiratory organs. Often, COPD patients suffer from exacerbations and comorbidities, such as chronic heart failure, diabetes or depression.

The WELCOME European project proposes an integrated approach offering, amongst other features, the possibility to closely monitor physiological parameters-which are indicative of the health status-in a patient's everyday environment. In this article, we present as contribution to the WELCOME project the design of a novel sensor-vest which allows recording a wide variety of body signals in high spatial and temporal resolution.

\section{A. Review}

Today, a plethora of 'smart garments', i.e., e-textiles with integrated sensors measuring different physiological signals, exists on the market. However, all of these devices share the same system structure: the sensors are embedded in the fabrics of a piece of clothing and connected to the electronics box the "hub" (comprising the microcontroller, the power supply and the transceiver) - placed in a pocket or plugged on the Tshirt.

As compared to bedside monitors that allow measuring many parameters, the number of sensors and measured signals in wearable systems is today limited. Amongst other reasons, this is due to complex cabling of the sensors requiring insulated, shielded, or even double-shielded wires as well as bulky
Barbara K. Pierscionek, Reem Kayyali, Shereen Elnabhani, Nada Philip

Kingston University

Kingston, United Kingdom

B.Pierscionek@kingston.ac.uk

The WELCOME Consortium*
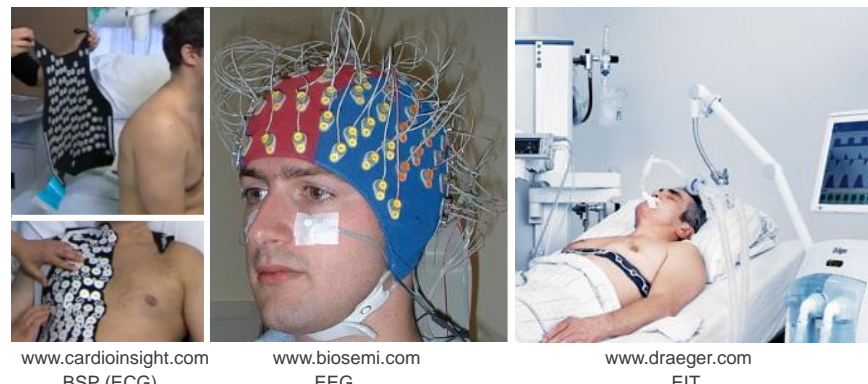

EEG

Fig. 1: Commercial bedside monitors for BSP (ECG), EEG, and EIT. connectors. Such cabling is undesired but acceptable for bedside monitors, whereas it is totally unacceptable for wearable systems. Examples of bedside monitors with a large number or signals are shown in Fig. 1. They include BSP (body surface potential) [1] or multi-lead ECG (electrocardiogram) [2], EEG (electroencephalogram) [3], and EIT (electro-impedance tomography).

\section{B. The SENSE approach (patented and commercialized $[4,5])$}

A first attempt to reduce cabling in ECG and impedance measurements using dry electrodes was done with the SENSE approach [6] where a single unshielded, possibly non-insulated electrical connection is used. This single electrical connection can be a conductive garment which connects two sensors and holds them against the body skin. Each sensor is standalone in the sense that each of them has its own battery and electronics. However, they are cooperative in the sense that both are needed to measure a one-lead ECG or a single impedance. No central hub is required for the functioning of the system.

In ECG mode (see Fig. 2), one sensor - the 'reference sensor'-contains two contacts with the body. One contact serves as a guard electrode and the other measures the voltage $u_{1}$. This voltage is controlled to zero with the controller $G$ acting on the voltage source $u_{0}$. As the voltage $u_{1}$ is controlled to zero, the ECG is directly measured as $u_{2}$ with the other sensor (called 'measuring sensor'). 


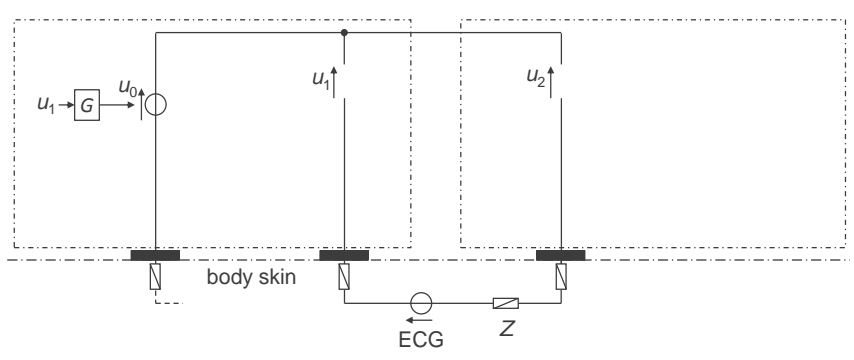

Fig. 2: Schematic circuit of two standalone sensors recording an ECG (patented SENSE approach). Left: reference sensor; right: measuring sensor.

This concept is however limited to one ECG lead. Adding more measuring sensors will provide more ECG leads (one per measuring sensor), but the signals would not be synchronized because each of them would be independently measured by its measuring sensor having its own time base (crystal).

When acquiring an impedance signal with the SENSE concept, the voltage $u_{1}$ is again controlled to zero in the reference sensor (see Fig. 3). The current $j_{3}$ is injected by the measuring sensor at a frequency which does not overlap with the ECG spectrum (typically about $50 \mathrm{kHz}$ ). This way, ECG and impedance can be measured together. The measuring sensor captures the voltage $u_{2}$ which is directly proportional to the impedance, since $j_{3}$ is a sine wave of constant magnitude. As both current injection and voltage measurement is performed in the same sensor, the frequency necessary to produce $j_{3}$ is readily available to demodulate $u_{2}$.

Note that the voltage between the inside of the body and the electrical connection linking the two cooperative sensors is $u_{1}$ which is controlled to zero. Therefore, in contrast to the classical approach that uses cables to inject currents requiring relatively high voltages (of the order of volts), the low voltage of the SENSE approach is of the order of fractions of millivolts. Therefore, the leakage current through the high impedance of the skin is negligible, even if the cable is not shielded or insulated.

While the SENSE approach allows reducing the cabling efforts, it has two main limitations. First, each SENSE sensor contains two electrodes that need to be at least separated by about $10 \mathrm{~mm}$. The minimum size of the sensor is directly linked to this distance. Second, the variation of the impedance of the electrical connection (e.g. due to variable stretch of conductive fabric) cannot be separated from bio-impedance measurements.

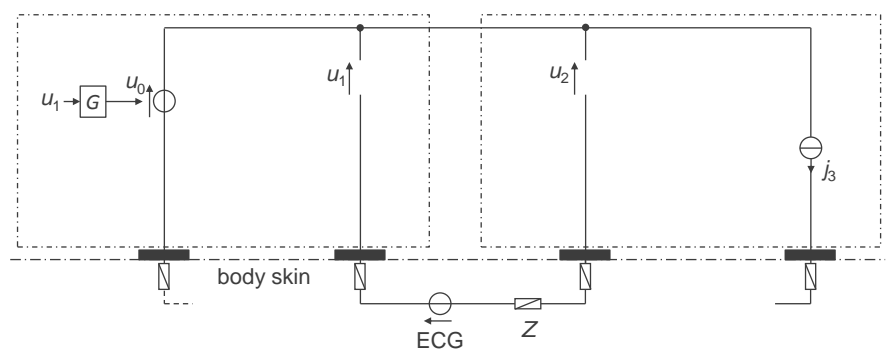

Fig. 3: Schematic circuit of two standalone sensors recording impedance $Z$ (patented SENSE approach). Left: reference sensor; right: measuring sensor.

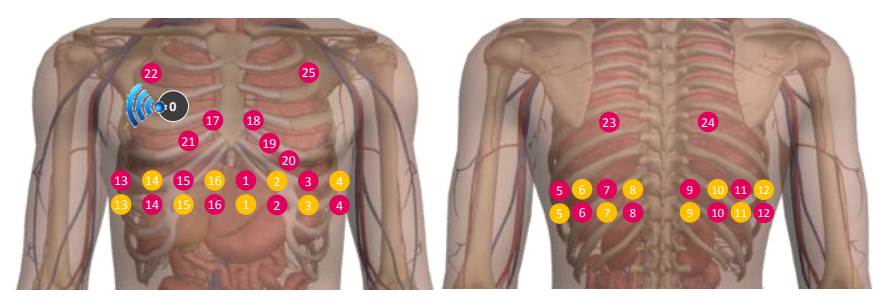

Fig. 4: Location of sensors in the WELCOME vest. Orange sensors inject EIT currents. Red sensors measure EIT voltages, ECG, and chest sounds.

The black sensor measures $\mathrm{SpO} 2$ and activity, synchronizes the other sensors, collects their data, records them,

\section{THE WELCOME APPROACH}

\section{A. Overview}

Extending the technology of the SENSE approach, in WELCOME, we are developing the technology for a truly wearable vest which measures in a high spatial and temporal resolution a wide variety of physiological signals, namely: EIT, chest sounds, ECG, SpO2, and body activity (see Fig. 4). Again, the WELCOME system relies on cooperative sensors and dry electrodes are employed for use comfort.

Fig. 5 shows the principle of ECG measurement with the WELCOME approach. Each sensor but the reference (guard) sensor measures a voltage $u_{i}$. In order to synchronously acquire these voltages $u_{i}$, all sensors must work in concert. Therefore, synchronization information is sent by the reference sensor to the measuring sensors.

The measured ECG voltages $u_{i}$ are transmitted to the reference sensor. The ECG values can therefore be stored or sent via wireless communication to the external world by the reference sensor. They are also used to control to zero their average or sum thanks to the controller $G$.

The WELCOME circuit for EIT measurement is shown in Fig. 6. The leftmost cooperative sensor still plays the role of a reference sensor. The other cooperative sensors are measuring sensors, but two types have to be distinguished, namely type I (injecting currents) and type V (measuring voltages). Both types have to be synchronized with respect to the reference sensor. The voltages measured by type $\mathrm{V}$ sensors are proportional to the bio-impedance, whereas the voltages measured across the current source of type I sensors are

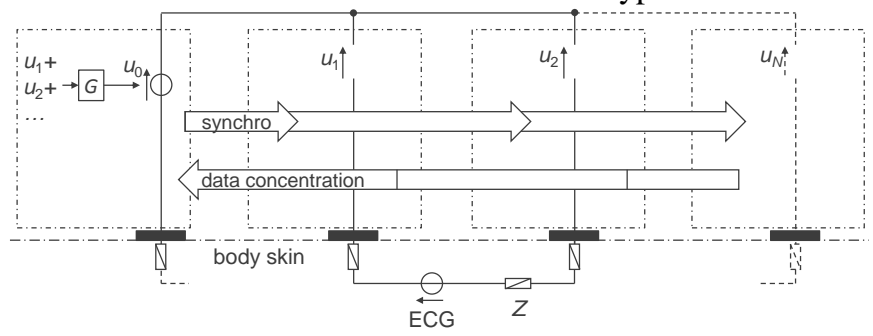

Fig. 5: Schematic circuit of cooperative sensors recording ECG signals (WELCOME approach, patent pending). The leftmost sensor is the 'reference sensor'; the others the 'measuring sensors'. 


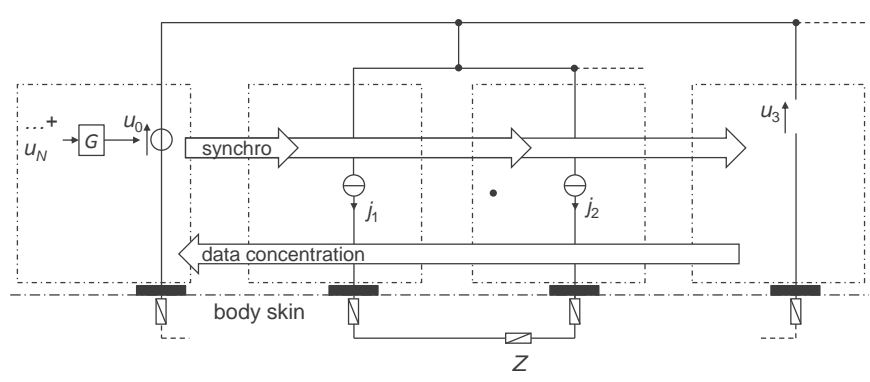

Fig. 6: Schematic circuit of cooperative sensors recording EIT signals (WELCOME approach, patent pending). The leftmost sensor is the 'reference sensor'; the others are the 'measuring sensors', some injecting a current (type I) or measuring a voltage (type V) proportional to bioimpedance $Z$.

proportional to the skin impedance (which may be good indicators of skin galvanic response or sweat).

As for the SENSE system, the WELCOME sensors are connected together with a single electrical connection that does not need to be shielded nor insulated.

However, in contrast to SENSE, the WELCOME vest measures several ECG leads and impedance channels in perfect synchronism. Moreover, variations of resistivity of the electrical connection no longer disturb the impedance measurement. Finally, as the cooperative sensors no longer have two contacts with the body but only one, their size can be reduced as it virtually only depends on the degree of integration.

\section{B. Electronics}

The synchronization and data concentration mechanisms of Fig. 5 and 6 can be performed with wireless technology. However, a wired approach is also possible as shown in Fig. 7. The voltage $u_{0}$ of the reference sensor carries the synchronization and control signals. A modulation at high frequency, like for instance at $2 \mathrm{MHz}$, is used not to interfere with the ECG (frequency band limited to a few hundred hertz) and EIT measurements (band about $50 \mathrm{kHz}$ ). These signals are picked up by the measuring sensors as voltage $u_{1}, \ldots, u_{N}$. More details about this mechanism are given in [7]. Likewise, the data are sent by the measuring sensors in the form of the current $j_{1}, \ldots, j_{N}$, each sensor using in turn a time slot. The data are received by the reference sensor with the current $j_{0}$.

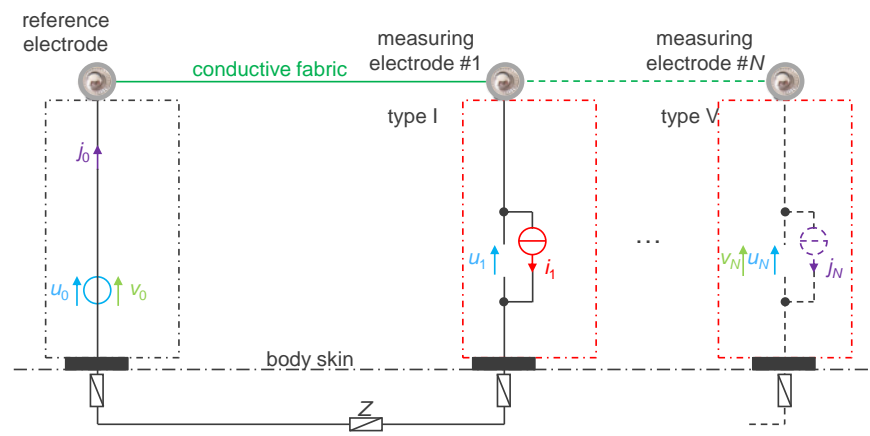

Fig. 7: Synchronization and control (light blue), data concentration (violet), ECG and impedance voltage measurement (light green), and impedance current injection (red) in the WELCOME vest.
The presented mechanisms have been tested using a first setup with one reference and two measurement sensors (see Fig. 8a). The sensors were integrated in a garment and connected to each other via an unshielded cable. In Fig. 8b), plots i) and ii) represent the raw ECG signals acquired by the two measurement sensors. The noise in these signals originates from an issue in this first setup that will be corrected in further developments. Since the same noise is seen by both measuring sensors, it is eliminated by computing the difference between the signals (shown in plot iii) of Fig. 8b), also demonstrating the perfect synchronism between the sensors.
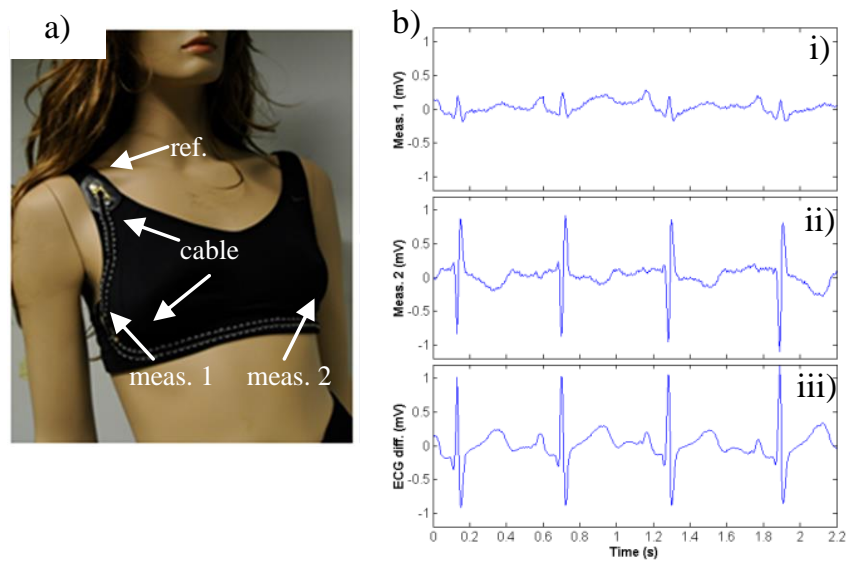

Fig. 8: a) Setup for testing the synchronization concept with one reference electrode and two measuring electrodes. The sensors are linked with an unshielded cable. b) Graphs i) and ii): Raw ECG signals from measurement electrode 1 and 2 with a small noise resulting from an issue in the first setup that will be corrected in futher developements. Graph iii): By computing the difference between signals i) and ii) the noise is eliminated.

\section{Housing of the sensors}

Requirements pertaining to the design of the sensor housings were found through a use case analysis and by evaluating a stakeholder survey carried out within the framework of the WELCOME project [9]:

- $\quad$ safety of the sensor under wet conditions

- removable attachment of the sensors to the vest

- connection between sensors through the vest

- cleaning of the sensors with an ethanol pad

- friendliness to the skin

- absorbance of mild mechanical shocks when worn

We satisfy all of the above requirements by encasing the sensor electronics inside a watertight housing (using sealing gaskets). In the proposed design (see Fig. 8), we favored compact, rounded geometries which conform to different body shapes. The proposed size of one sensor is $35 \mathrm{~mm}$ in diameter and $10 \mathrm{~mm}$ in height. Fig. 8 also shows that the sensor housings are equipped with snap buttons. Their function is to attach the sensors to the vest in a removable manner and to connect them electrically to the vest electrical connection. The sensor housing is made of biocompatible materials, such as surgical grade stainless steel (metallic parts in contact with the skin), silicone and hard plastics which are approved for use in medical devices (e.g., polyamide, polyurethane, etc.).

While type I sensors contain a simple metal electrode which is in contact with the skin, type $\mathrm{V}$ sensors are 


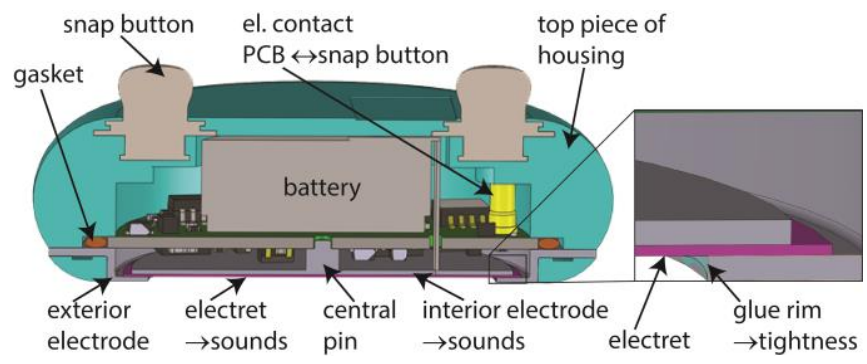

Fig. 9: Section view of a type-V sensor containing an annular (exterior) electrode, an electret, and an interior electrode for chest sound recording. additionally equipped with an electret foil which transduces the chest sounds. The electret foil is coated on both sides with a silver film and sits on the inside of the exterior electrode which in the type $\mathrm{V}$ configuration has a hole in the centre. This way, the electret and the electrode contact the skin concurrently.

To guarantee water tightness of the type $\mathrm{V}$ sensors, the electret is glued to the hole in the exterior electrode (see inset in Fig. 8). Since the measuring principle of an electret is a force-dependent voltage difference between the two sides of the sheet, both sides of the electret need to be electrically contacted. In the present design, the voltage difference between the side touching the skin and the opposite (interior) side is assessed via the aforementioned exterior electrode and an interior round electrode (see Fig. 8). The interior electrode is held in place by the exterior electrode (without touching the latter) and the PCB to which it is connected via a central pin. The central pin also ensures the electrical contact between the PCB and the electret.

To verify the geometrical design of the housing and the assembly, relevant parts were manufactured by fused deposition modelling and machining (see Fig 10 a). In addition, the ergonomics of the housing were tested using 3D printed mock-ups of the casing of the sensors which were attached to the interior of a tight-fitting vest (see Fig. 10 b). In summary, the design of the sensors allows for high wearing comfort, while providing sufficient contact between the sensors and the skin.

\section{CONCLUSION}

In conclusion, we have proposed and discussed a vest which measures various physiological signals using embedded
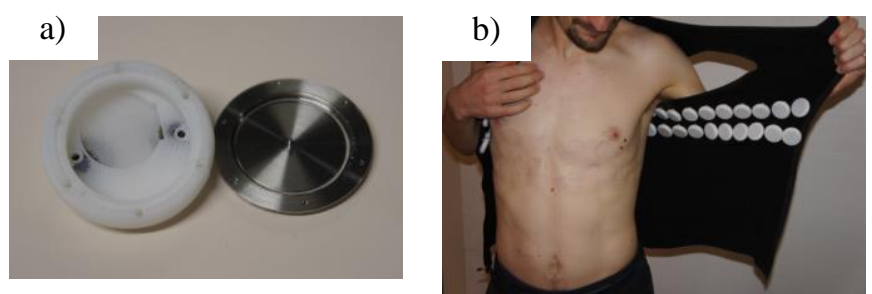

Figure 10: a) Prototypes of the top piece of the housing and the electrodes were used to verify that the parts can be assembled correctly. b) The wearing comfort and the contact pressure of the sensors on the skin were assessed using $3 \mathrm{D}$ printed mockups integrated in a tight fitting vest. cooperative sensors. The communication and synchronization of the sensors is guaranteed using a simple electrical connection. The reduced complexity of the design facilitates the manufacturing of the WELCOME vest and will make it a pleasurable experience to wear for patients.

\section{ACKNOWLEDGMENT}

This work was supported by the European commission (Call Identifier FP7-ICT-2013-10).

*The WELCOME consortium consists of Exus, Centre Suisse d'Electronique et de Microtechnique, Kingston University London, Aristotle University of Thessaloniki, Inventya Ltd, Christian-Albrechts-Universität zu Kiel, Universitätsklinikum Schleswig-Holstein, Royal College of Surgeons in Ireland, Smartex S.R.L., Ciro+ B.V., Kristronics GmbH, Universidade de Coimbra and Croydon Healthcare NHS Trust.

\section{REFERENCES}

[1] Y. Rudy, "Noninvasive Electrocardiographic Imaging in Humans" J Electrocardiol 2005;38:138-140.

[2] A.C. MettingVanRijn, A. Peper, C.A. Grimbergen, "High-quality recording of bioelectric events, Part 1 Interference reduction, theory and practice”, Med. \& Biol. Eng. \& Comput, Volume 28, 1990, pp 389-397.

[3] A.C. MettingVanRijn, A.P. Kuiper, T.E. Dankers, C.A. Grimbergen, "Low-cost active electrode improves the resolution in biopotential recordings", Proceedings of the $18^{\text {th }}$ annual international conference of the IEEE Volume 1, 1996, pp 101-102.

[4] O. Chételat, J. Oster, O. Grossenbacher, A. Hutter, J. Krauss, and A. Giannakis, "A Highly Integrated Wearable Multi-parameter Monitoring System for Athletes", IFMBE Proceedings Volume 34, 2011, pp 148$151,15^{\text {th }}$ Nordic-Baltic Conference on Biomedical Engineering and Medical Physics (NBC 2011).

[5] www.senseyourcore.com

[6] O. Chételat, R. Gentsch, J. Krauss, and J. Luprano, "Getting rid of the wires and connectors in physiological monitoring", Conf Proc IEEE Eng Med Biol Soc. 2008; 2008:1278-82.

[7] O. Chételat, R. Schmid, O. Grossenbacher, M. Rapin, J-A. Porchet, C. Meier, R. Abächerli, "Standalone dry electrode-sensors for multilead ECG monitoring in mobile patients", ESGCO Proceedings, 2014, $8^{\text {th }}$ Conference of the European study group on cardiovascular oscillations.

[8] S. Nabhani-Gebara, R. Kayyali, The WELCOME Consortium, "WELCOME project: What do stakeholders want?", MobiHealth Conference, 2014. 